\title{
COMMUTING PROJECTIONS WITH ASSIGNED RANGES
}

\section{TAKAGUCHI}

1. Let $\mathfrak{W}$ be a Hilbert space. A projection is a bounded idempotent linear operator. Orthogonal projections form a proper subclass of the class of all projections. A problem is to get a condition for there to exist commuting projections $E_{1}, \cdots, E_{n}$ with the same ranges as given orthogonal projections $P_{1}, \cdots, P_{n}$ respectively. This will be settled in terms of properties of the sublattice, generated by $P_{1}$, $\cdots, P_{n}$ in the lattice of all orthogonal projections. In case $n=2$, commuting projections with minimum norms are constructed. Another problem is to find commuting projections $E_{1}, \cdots, E_{n}$ in a Hilbert space $\Omega$, containing $\mathfrak{S}$ as a subspace, such that $P_{j} x=P E_{j} x$ for $x \in \mathfrak{S}, j=1,2, \cdots, n$, where $P$ is the orthogonal projection from $\Omega$ on to $\mathfrak{G}$. This will be proved to be always possible.

2. Let $P$ and $Q$ be orthogonal projections with ranges $\mathfrak{M}$ and $\mathfrak{N}$ respectively. Then $P \wedge Q$ and $P \vee Q$ will denote the orthogonal projections on to $\mathfrak{M} \cap \mathfrak{N}$ and the closure of $\mathfrak{M}+\mathfrak{N}$ respectively. $\mathfrak{M} \ominus \mathfrak{N}$ will stand for the subspace $\mathfrak{M} \cap(\mathfrak{M} \cap \mathfrak{R})^{\perp}$ and $P \ominus Q$ is the orthogonal projection on to it.

If there exist commuting projections $E$ and $F$ with ranges $\mathfrak{M}$ and $\mathfrak{N}$ respectively, $\mathfrak{M}+\mathfrak{N}$ becomes the range of the projection $E+F$ $-E F$, therefore it is a closed subspace.

If, conversely, $\mathfrak{M}+\mathfrak{N}$ is closed, in view of the well-known theorem of Kober (see [3]) the operators $E^{\prime}$ and $F^{\prime}$, which assign to each $x \in \mathfrak{M}+\mathfrak{R}$ the elements $u+v$ and $u+w$ respectively, are continuous, where $u, v$ and $w$ are uniquely determined by the relation $x=u+v+w$ with $u \in \mathfrak{M} \cap \mathfrak{N}, v \in \mathfrak{M} \ominus \mathfrak{N}$ and $w \in \mathfrak{N} \ominus \mathfrak{M}$. Then the operators $E$ $=E^{\prime}(P \vee Q)$ and $F=F^{\prime}(P \vee Q)$ are commuting projections with ranges $\mathfrak{M}$ and $\mathfrak{R}$ respectively.

It was shown by Mackey $[3$, p. 166] that the closedness of $\mathfrak{M}+\mathfrak{R}$ is equivalent to the property that $(P \vee Q) \wedge Q^{\prime}=\left(P \wedge Q^{\prime}\right) \vee Q$ for every orthogonal projection $Q^{\prime}$ with $Q \leqq Q^{\prime}$.

Suppose again that there exist commuting projections $E$ and $F$ with ranges $\mathfrak{M}$ and $\mathfrak{N}$ respectively. Since the commutativity implies that $E \mathfrak{R} \subseteq \mathfrak{M} \cap \mathfrak{N}$ for any $x, y \in \mathfrak{W},(P \ominus Q) x$ and $E Q y$ are orthogonal, therefore

$$
\|(P \ominus Q) x\|^{2} \leqq\|(P \ominus Q) x-E Q y\|^{2} \leqq\|E\|^{2} \cdot\|(P \ominus Q) x-Q y\|^{2} .
$$

Received by the editors May 15, 1967. 
Since, for fixed $x$, the minimum of $\|(P \ominus Q) x-Q y\|^{2}, y \in \mathfrak{W}$ is equal to $\|(P \ominus Q) x\|^{2}-\|Q(P \ominus Q) x\|^{2}$, it follows that

$$
\|Q(P \ominus Q) x\|^{2} \leqq\left\{1-\|E\|^{-2}\right\} \cdot\|(P \ominus Q) x\|^{2},
$$

consequently

$$
\|(Q \ominus P)(P \ominus Q)\|^{2} \leqq 1-\|E\|^{-2}<1,
$$

because

$$
(Q \ominus P)(P \ominus Q)=Q(P \ominus Q) .
$$

Suppose, conversely, that $\|(Q \ominus P)(P \ominus Q)\|<1$. Then both $I$ $-(Q \ominus P)(P \ominus Q)$ and $I-(P \ominus Q)(Q \ominus P)$ have bounded inverses and the operators

$$
E_{0}=P_{\wedge} Q+(P \ominus Q)\{I-(Q \ominus P)(P \ominus Q)\}^{-1} \cdot(I-Q \ominus P)
$$

and

$$
F_{0}=P_{\wedge} Q+(Q \ominus P)\{I-(P \ominus Q)(Q \ominus P)\}^{-1} \cdot(I-P \ominus Q)
$$

are commuting projections with ranges $\mathfrak{N}$ and $\mathfrak{N}$ respectively. This can be seen by using the expansion

$$
\{I-(Q \ominus P)(P \ominus Q)\}^{-1}=\sum_{n=0}^{\infty}[(Q \ominus P)(P \ominus Q)]^{n} .
$$

The definition shows that $E_{0} F_{0}=P \wedge Q$ and $E_{0}+F_{0}-E_{0} F_{0}=P \vee Q$ and that $E_{0}\left(I-F_{0}\right)$ and $F_{0}\left(I-E_{0}\right)$ have the same ranges as $P \ominus Q$ and $Q \ominus P$ respectively. Then it follows that

$$
\begin{aligned}
\|x\|^{2}-\left\|E_{0} F_{0} x\right\|^{2} & \geqq\|(P \vee Q-P \wedge Q) x\|^{2} \\
& =\left\|E_{0}\left(I-F_{0}\right) x+F_{0}\left(I-E_{0}\right) x\right\|^{2} \\
& \geqq\left\{1-\|(Q \ominus P)(P \ominus Q)\|^{2}\right\} \cdot\left\|E_{0}\left(I-F_{0}\right) x\right\|^{2},
\end{aligned}
$$

therefore

$$
\|x\|^{2} \geqq\left\{1-\|(Q \ominus P)(P \ominus Q)\|^{2}\right\}\left\|E_{0} x\right\|^{2},
$$

which, together with the inequality already obtained, implies that

$$
\left\|E_{0}\right\|=\left\{1-\|(Q \ominus P)(P \ominus Q)\|^{2}\right\}^{-1 / 2} .
$$

The norm of $F_{0}$ is shown to have the same value.

It is desired to give an expression for the quantity $\|(Q \ominus P)(P \ominus Q)\|$ in terms of $P \vee Q$ and $P \wedge Q$.

LEMMA. $\|(Q \ominus P)(P \ominus Q)\|=\|P \vee Q+P \wedge Q-P-Q\|$. 
Proof. Since

$P \vee Q+P \wedge Q-P-Q=(P \ominus Q) \vee(Q \ominus P)-(P \ominus Q)-(Q \ominus P)$ and $(P \ominus Q) \wedge(Q \ominus P)=0$, it suffices to prove $\|I-P-Q\|=\|Q P\|$ under the assumption that $P \wedge Q=0$ and $P+Q=I$.

Since it is known $[1$, p. 70$]$ that

$$
\|I-P-Q\|=\operatorname{Max}\{\|Q P\|,\|(I-Q)(I-P)\|\},
$$

the assertion is true in case $\|Q P\|=1$. If $\|Q P\|<1$, in view of the foregoing result there exist the unique commuting projections $E$ and $F$ with the same ranges as $P$ and $Q$ respectively and their norms are equal to $\left\{1-\|Q P\|^{2}\right\}^{-1}$. On the other hand, $E^{*}$ and $F^{*}$ are the unique commuting projections with the same ranges as $I-Q$ and $I-P$ respectively. The same discussion is applied to get

$$
\|E\|=\left\|E^{*}\right\|=\left\{1-\|(I-P)(I-Q)\|^{2}\right\}^{1 / 2},
$$

therefore

$$
\|(I-P)(I-Q)\|=\|Q P\| .
$$

Use, finally, the relation

$$
\|(I-P)(I-Q)\|=\|(I-Q)(I-P)\| .
$$

In view of this lemma, the preceding results can be summarized in the following theorem.

Theorem 1. For a pair of orthogonal projections $P, Q$ the following conditions are mutually equivalent:

(1) there exist commuting projections $E$ and $F$ with the same ranges as $P$ and $Q$ respectively;

(2) $(P \vee Q) \wedge Q^{\prime}=\left(P \wedge Q^{\prime}\right) \vee Q$ for every orthogonal projection $Q^{\prime}$ with $Q \leqq Q^{\prime}$

(3) $\|P \vee Q+P \wedge Q-P-Q\|<1$. When one (and all) of these conditions is fulfilled, there exist such commuting projections $E_{0}$ and $F_{0}$ with minimum norm:

$$
\left\|E_{0}\right\|=\left\|F_{0}\right\|=\left\{1-\|P \vee Q+P \wedge Q-P-Q\|^{2}\right\}^{-1 / 2} .
$$

A related problem is to ask when there exist doubly commuting projections $E$ and $F$, i.e. $E F=F E$ and $E * F=F E^{*}$, with the same ranges as $P$ and $Q$ respectively. The answer is, however, quite simple. It is the case (when and) only when $P Q=Q P$. In fact, since the range of the projection $I-E^{*}$ is the orthogonal complement of the range of $E$ and is invariant under $F$ by the double commutativity, the range 
of $E$ reduces $F$, i.e. $F P=P F$. A similar argument yields $P Q=Q P$.

3. Let $P_{1}, \cdots, P_{n}$ be orthogonal projections with ranges $\mathfrak{M}_{1}$, $\cdots, \mathfrak{M}_{n}$ respectively. Consider the sublattice $L\left(P_{1}, \cdots, P_{n}\right)$, generated by $P_{1}, \cdots, P_{n}$ in the lattice of all orthogonal projections, and the sublattice $L\left(\mathfrak{M}_{1}, \cdots, \mathfrak{M}_{n}\right)$, generated by $\mathfrak{M}_{1}, \cdots, \mathfrak{M}_{n}$ in the lattice of all (not necessarily closed) subspaces. If the lattice $L\left(\mathfrak{M}_{1}, \cdots, \mathfrak{M}_{n}\right)$ consists of closed subspaces only, it is isomorphic to the lattice $L\left(P_{1}, \cdots, P_{n}\right)$.

Theorem 2. For orthogonal projections $P_{1}, \cdots, P_{n}$ the following conditions are equivalent:

(1) there exist commuting projections $E_{1}, \cdots, E_{n}$ with the same ranges as $P_{1}, \cdots, P_{n}$ respectively;

(2) the sublattice $L\left(P_{1}, \cdots, P_{n}\right)$, generated by $P_{1}, \cdots, P_{n}$ in the lattice of all orthogonal projections, is distributive and possesses one (and both) of the following equivalent properties:

(a) $\|P \vee Q+P \wedge Q-P-Q\|<1$ for $P, Q \in L\left(P_{1}, \cdots, P_{n}\right)$,

(b) $(P \vee Q) \wedge Q^{\prime}=\left(P \wedge Q^{\prime}\right) \vee Q$ for $P, Q \in L\left(P_{1}, \cdots, P_{n}\right)$ and for every orthogonal projection $Q^{\prime}$ with $Q \leqq Q^{\prime}$.

Proof. Suppose that the condition (1) is fulfilled, then the lattice $L\left(\mathfrak{M}_{1}, \cdots, \mathfrak{M}_{n}\right)$ is distributive, where $\mathfrak{M}_{j}$ is the range of $P_{j}, j=1,2$, $\cdots, n$. In fact, for any three members $\mathfrak{N}_{1}, \mathfrak{N}_{2}, \mathfrak{N}_{3}$ in it there exist commuting projections $F_{1}, F_{2}, F_{3}$, which are polynomials of $E_{1}$, $\cdots, E_{n}$ and have ranges $\mathfrak{N}_{1}, \mathfrak{N}_{2}, \mathfrak{R}_{3}$ respectively. Since $\left(\mathfrak{R}_{1}+\mathfrak{R}_{2}\right) \cap \mathfrak{N}_{3}$ becomes the range of the projection $F_{1}\left(I-F_{2}\right) F_{3}+F_{2} F_{3}$, it follows that

$$
\left(\mathfrak{R}_{1}+\mathfrak{N}_{2}\right) \cap \mathfrak{R}_{3}=\left(\mathfrak{R}_{1} \cap \mathfrak{R}_{3}\right)+\left(\mathfrak{R}_{2} \cap \mathfrak{N}_{3}\right) .
$$

Further every member of $L\left(\mathfrak{M}_{1}, \cdots, \mathfrak{M}_{n}\right)$ is a closed subspace, because it is a range of a projection. Then in view of Theorem 1 and the comment preceding Theorem 2 , the lattice $L\left(P_{1}, \cdots, P_{n}\right)$, which becomes isomorphic to the lattice $L\left(\mathfrak{M}_{1}, \cdots, \mathfrak{M}_{n}\right)$, is distributive and possesses the properties (a) and (b).

Suppose, conversely, that the condition (2) is fulfilled. Then as in the above discussion, the lattice $L\left(\mathfrak{M}_{1}, \cdots, \mathfrak{M}_{n}\right)$ is distributive and consists of closed subspaces only. Now let $J_{k}$ (or $L_{k}$ ) denote generally a set, consisting of $k$ integers in $\{1,2, \cdots, n\}$. Put for $k$ and $J_{k}$

$$
\mathfrak{M}\left(J_{k}\right)=\bigcap_{j \in J_{k}} \mathfrak{M}_{j}, \quad \mathfrak{M}^{(k)}=\sum_{\mathrm{al1}} \mathfrak{M}\left(J_{k}\right)
$$

and

$$
\mathfrak{N}\left(J_{k}\right)=\mathfrak{M}\left(J_{k}\right) \ominus \mathfrak{M}^{(k+1)}, \quad\left(\mathfrak{M}^{(n+1)}=\{0\}\right) .
$$


Let us prove by induction that

$$
\mathfrak{N}_{j}=\sum_{k=1}^{n} \sum_{j \in J_{k}} \mathfrak{N}\left(J_{k}\right), \quad j=1,2, \cdots, n .
$$

This is trivial for $n=1$. Assuming that the assertion is generally true for $n-1$, consider the subspaces $\mathfrak{M}_{j}^{*}=\mathfrak{M}_{j} \cap \mathfrak{M}_{n}, j=1,2, \cdots$, $n-1$ and define subspaces $\mathfrak{M}^{*}\left(J_{k}\right), \mathfrak{M}^{*(k)}$ and $\mathfrak{N}^{*}\left(J_{k}\right)$ (with $J_{k}$ $C\{1,2, \cdots, n-1\})$ from $\mathfrak{M}_{1}^{*}, \cdots, \mathfrak{M}_{n-1}^{*}$ just as $\mathfrak{M}\left(J_{k}\right), \mathfrak{M}^{(k)}$ and $\mathfrak{N}\left(J_{k}\right)$ were defined from $\mathfrak{M}_{1}, \cdots, \mathfrak{M}_{n}$. Then the distributivity shows that

$$
\mathfrak{M}^{*(k)}=\sum_{J_{k}}\left[\mathfrak{M}_{n} \cap \mathfrak{M}\left(J_{k}\right)\right]=\mathfrak{M}_{n} \cap \mathfrak{M}^{(k+1)} ;
$$

hence $\mathfrak{R}^{*}\left(J_{k}\right)=\mathfrak{R}\left(J_{k}, n\right)$. Since the property, in question, of $L\left(\mathfrak{M}_{1}, \cdots, \mathfrak{M}_{n}\right)$ implies that the lattice $L\left(\mathfrak{M}_{1}^{*}, \cdots, \mathfrak{M}_{n-1}^{*}\right)$ is distributive and consists of closed subspaces only, it follows from the induction assumption that

$$
\mathfrak{M}_{j}^{*}=\sum_{k=1}^{n} \sum_{j \in J_{k}} \mathfrak{R}^{*}\left(J_{k}\right), \quad j=1,2, \cdots, n-1 .
$$

But this can be written in the form

$$
\mathfrak{M}_{j} \cap \mathfrak{M}_{n}=\sum_{k=2}^{n} \sum_{j, n \in J_{k}} \mathfrak{N}\left(J_{k}\right) .
$$

A similar argument proves that for any distinct $i, j$

$$
\mathfrak{M}_{j} \cap \mathfrak{M}_{i}=\sum_{k=2}^{n} \sum_{i, j \in J_{k}} \mathfrak{N}\left(J_{k}\right) .
$$

Since for any $j$ the distributivity implies that

$$
\mathfrak{M}_{j}=\mathfrak{N}(j)+\mathfrak{M}_{j} \cap \mathfrak{M}^{(2)}=\mathfrak{N}(j)+\sum_{i \neq j}\left(\mathfrak{M}_{j} \cap \mathfrak{M}_{i}\right),
$$

the above result yields the required expression for $\mathfrak{M}_{j}$.

Next let us prove linear independence of the family of all nontrivial $\mathfrak{N}\left(J_{k}\right)$ 's. To this end. it suffices to prove that for any $J_{k}$

$$
\mathfrak{N}\left(J_{k}\right) \cap\left\{\sum_{L_{k \neq J}} \mathfrak{N}\left(L_{k}\right)+\mathfrak{M}^{(k+1)}\right\}=\{0\},
$$

because $\mathfrak{N}\left(J_{i}\right) \subseteq \mathfrak{M}^{(k+1)}$ whenever $k<i$. The assertion results from the following relations, based on the distributivity 


$$
\begin{aligned}
\mathfrak{N}\left(J_{k}\right) & \cap\left\{\sum_{L_{k} \neq J_{k}} \mathfrak{N}\left(L_{k}\right)+\mathfrak{M}^{(k+1)}\right\} \subseteq \mathfrak{M}\left(J_{k}\right) \\
& \cap\left\{\sum_{L_{k} \neq J_{k}} \mathfrak{M}\left(L_{k}\right)+\mathfrak{M}^{(k+1)}\right\}=\mathfrak{M}\left(J_{k}\right) \cap \mathfrak{M}^{(k+1)} .
\end{aligned}
$$

Now since $\mathfrak{N}\left(J_{k}\right)$ 's have the closed sum $\mathfrak{M}^{(1)}$, in view of Kober's theorem there exist mutually annihilating projections $E\left(J_{k}\right)$ with ranges $\mathfrak{N}\left(J_{k}\right)$ respectively. Then the operators

$$
E_{j}=\sum_{k=1}^{n} \sum_{j \in J_{k}} E\left(J_{k}\right), \quad j=1,2, \cdots, n,
$$

are commuting projections with ranges $\mathfrak{M}_{j}$. This completes the proof.

Corollary. Let each of two families of orthogonal projections $\left\{P_{1}, \cdots, P_{n}\right\}$ and $\left\{Q_{1}, \cdots, Q_{m}\right\}$ satisfy the condition in Theorem 2. If each $P_{i}$ commutes with all $Q_{j}$, then the combined family $\left\{P_{1}, \cdots, P_{n}\right.$, $\left.Q_{1}, \cdots, Q_{m}\right\}$ satisfies the same condition, too.

Proof. With the same notations as in the proof of Theorem 2, each subspace in $L\left(\mathfrak{M}_{1}, \cdots, \mathfrak{M}_{n}\right)$ is invariant under all $Q_{j}$, hence so is each $\mathfrak{N}\left(J_{k}\right)$, which leads to the commutativity of each $E_{i}$ with all $Q_{j}$. Similar argument shows that there exist commuting projections $F_{1}, \cdots, F_{m}$ which have the same ranges as $Q_{1}, \cdots, Q_{m}$ respectively and commute with all $E_{i}$.

4. When $\mathfrak{S}$ is imbedded in a larger Hilbert space $\Omega$, a linear operator $S$ in $\Omega$ is called a dilation of a linear operator $T$ in $\mathfrak{W}$, in case $S x=P T x$ for $x \in \mathfrak{W}$, where $P$ is the orthogonal projection from $\Omega$ onto $\mathfrak{S}$.

In a previous paper [2] we proved that if a pair of orthogonal projections admits dilations which are commuting orthogonal projections, then they necessarily commute with each other. In this connection the following theorem is of interest.

Theorem 3. Orthogonal projections $P_{1}, \cdots, P_{n}$ admit dilations $E_{1}, \cdots, E_{n}$, which are commuting projections.

Proof. Consider the orthogonal sum

$$
\Re=\mathfrak{S}_{\mathfrak{Q}} \oplus \mathfrak{S}_{1} \oplus \cdots \oplus \mathfrak{W}_{n}
$$

where each $\mathfrak{W}_{j}$ is a copy of $\mathfrak{S}$. Imbed $\mathfrak{S}$ and $\mathfrak{S}_{j}$ canonically into $\Omega$. The isomorphism from $\mathfrak{S}$ to $\mathfrak{S}_{j}$ will be denoted by $I_{j}$. Consider $n+1$ subspaces defined by 


$$
\mathfrak{N}_{j}=\left\{\left(P_{j}+I_{j} P_{j}\right) x ; x \in \mathfrak{S}\right\}, \quad j=1,2, \cdots, n
$$

and

$$
\mathfrak{l}=\left\{\left(I-\sum_{j=1}^{n} P_{j}-\sum_{j=1}^{n} I_{j} P_{j}\right) x ; x \in \mathfrak{W}\right\} .
$$

Then it is easy to see that these subspaces are linearly independent with a closed sum; therefore in view of Kober's theorem there exist mutually annihilating projections $E_{1}, \cdots, E_{n}$ with ranges $\mathfrak{R}_{1}$, $\cdots, \mathfrak{N}_{n}$ respectively. Since each $x \in \mathfrak{W}$ is written in the form

$$
x=\sum_{j=1}^{n}\left(P_{j}+I_{j} P_{j}\right) x+\left(I-\sum_{j=1}^{n} P_{j}-\sum_{j=1}^{n} I_{j} P_{j}\right) x,
$$

it follows that $E_{j} x=P_{j} x+I_{j} P_{j} x$, which means that $E_{j}$ is a dilation of $P_{j}$.

The author wishes to express his gratitude to Professor T. Ando for his valuable advice.

\section{REFERENCES}

1. N. I. Akhiezer and I. M. Glazman, Theory of linear operators in Hilbert space, Ungar, New York, 1961.

2. T. Ando and M. Takaguchi, Commuting dilations of self-adjoint operators, Proc. Japan Acad. 41 (1965), 696-698.

3. G. Mackey, On infinite-dimensional linear spaces, Trans. Amer. Math. Soc. 57 (1945), 155-207.

Hokkaido University, Sapporo, Japan 